\title{
Polarization Insensitive, Broadband, Near Diffraction-Limited Metalens in Ultraviolet Region
}

\author{
Saima Kanwal ${ }^{1}$, Jing Wen ${ }^{1}$, Binbin Yu ${ }^{1}$, Xu Chen ${ }^{1}$, Dileep Kumar ${ }^{2}$, Yi Kang ${ }^{1}$, Chunyan Bai ${ }^{1}$, \\ Saima Ubaid ${ }^{1}$ and Dawei Zhang ${ }^{1,3, *}$ \\ 1 Engineering Research Center of Optical Instrument and Systems, Ministry of Education and Shanghai Key \\ Lab of Modern Optical System, University of Shanghai for Science and Technology, No. 516 Jun Gong Road, \\ Shanghai 200093, China; 142499021@st.usst.edu.cn (S.K.); jwen@usst.edu.cn (J.W.); \\ 161390013@st.usst.edu.cn (B.Y.); 191380026@st.usst.edu.cn (X.C.); 171390035@st.usst.edu.cn (Y.K.); \\ 171390036@st.usst.edu.cn (C.B.); 161399008@st.usst.edu.cn (S.U.) \\ 2 State Key Laboratory of Industrial Control Technology, College of Control Science and Engineering, \\ Zhejiang University, Hangzhou 310027, China; 11332042@zju.edu.cn \\ 3 Shanghai Institute of Intelligent Science and Technology, Tongji University, Shanghai 200093, China \\ * Correspondence: dwzhang@usst.edu.cn
}

Received: 24 June 2020; Accepted: 16 July 2020; Published: 23 July 2020

\begin{abstract}
Metasurfaces in the ultraviolet spectrum have stirred up prevalent research interest due to the increasing demand for ultra-compact and wearable UV optical systems. The limitations of conventional plasmonic metasurfaces operating in transmission mode can be overcome by using a suitable dielectric material. A metalens holds promising wavefront engineering for various applications. Metalenses have developed a breakthrough technology in the advancement of integrated and miniaturized optical devices. However, metalenses utilizing the Pancharatnam-Berry (PB) phase or resonance tuning methodology are restricted to polarization dependence and for various applications, polarization-insensitive metalenses are highly desirable. We propose the design of a high-efficiency dielectric polarization-insensitive UV metalens utilizing cylindrical nanopillars with strong focusing ability, providing full phase delay in a broadband range of Ultraviolet light (270-380 nm). The designed metalens comprises Silicon nitride cylindrical nanopillars with spatially varying radii and offers outstanding polarization-insensitive operation in the broadband UV spectrum. It will significantly promote and boost the integration and miniaturization of the UV photonic devices by overcoming the use of Plasmonics structures that are vulnerable to the absorption and ohmic losses of the metals. The focusing efficiency of the designed metalens is as high as $40 \%$.
\end{abstract}

Keywords: UV; metalens; polarization insensitive; broadband; dielectric; diffraction-limited

\section{Introduction}

Ultraviolet light plays a vital part in human life involving sterilizers, lithography equipment, and laser devices. Lenses are one of the essential components among the UV equipment for convergence of UV light. Traditional optical components and lenses use the refractive optical scheme i.e., gradual phase accumulation for the wavefront shaping during the propagation of the light which results in expensive, bulky, and low-efficiency lenses [1]. On the other hand, metasurfaces that are ultra-thin optical structures have appeared to provide a substantial way, by discrete phase changes over the subwavelength scale, to control the amplitude, phase and polarization of the light [2-4] to realize optical functionalities by their planar structure [5] such as meta-holograms [6], axicons metalenses [7], antireflection coatings color filters [8], color imaging [9,10], optical vortex generators and polarizers [11,12] and numerous novel photonic devices and systems [13]. Metasurfaces have 
gained substantial interest in the past few years [14,15] in having the ability to replace the bulky, expensive, low-efficiency optical components $[7,16]$ and provide a gateway towards the miniaturization of the optical devices and components $[17,18]$. Moreover, metasurfaces are greatly desired in integrated optics and electronic circuits, portals, and mobile devices. They are attaining a great interest in industrial applications due to their high efficiency of manipulating the light $[19,20]$.

Electromagnetic phase control is the most distinctive and perhaps the simplest application of the metasurfaces [21]. The key to implementing various applications is the control of the full $2 \pi$ phase. There are numerous ways to modulate the phase of the electromagnetic waves in a convenient manner, such as geometrical phase control and dynamical phase control. Geometrical phase metasurfaces that are proposed and experimentally demonstrated, utilize the employment of a dominant electrical resonance by asymmetric nanopillars to induce local phase retardation by rotating the polarization of incident light. This sort of phase retardation, that is also called the Pancharatnam-Berry (PB) phase shift, can be employed for beam shaping and spin filter applications. Employing this method, efficiency as high as $86 \%$ is validated experimentally for lensing. Transmissive metasurfaces designed by the PB phase method have been demonstrated in IR and visible range with anomalous properties, but this method is specifically restricted to circularly polarized incident light. The second approach that is used to modulate the phase of light is dynamical phase control, which is independent of the polarization of the incident light. This approach attains the desired optical path difference by changing the optical path of the light [22]. Interfacial phase discontinuities are introduced by the metasurfaces along the optical path as an alternative approach to realize the flat and compact dynamical phase elements

Plasmonic metasurfaces and lenses have a very low efficiency equated to the dielectric metasurfaces due to the high inherent Joule losses at optical frequencies, particularly in transmissive mode [23-25]. The energy efficiency of these systems is no higher than $20 \%$. Besides, metals are not stable at higher temperatures, and so they are not an appropriate option for high power laser applications. However, these losses can be reduced by adopting a dielectric material having a high refractive index, a larger bandgap, and lower absorption losses at the optical frequencies [26].

Depending on the optical properties of the material, previous work on metalenses has focused on diverse wavelengths ranging from Ultraviolet to near-infrared [27]. However, due to insufficient phase delay, these lenses defocused at a short UV wavelength range [28-32], and these do not operate in the broadband UV spectrum. Moreover, they do not support the polarization independent operation of the reported metalens $[28,33,34]$.

We have recently demonstrated a Pancharatnam-Berry phase-based high-efficiency broadband metalens in the UV spectrum (250-400 nm), which requires circularly polarized (CP) incident light [35]. In this article, we demonstrate the design of a polarization insensitive, transmissive planar metasurface lens composed of silicon nitride $\mathrm{Si}_{3} \mathrm{~N}_{4}$ cylindrical nanopillars operating at the broadband UV range (270-380 nm). By varying the radius of the nanopillar abrupt phase, changes can be realized. A flat metasurface lens operating in broadband UV spectrum with corresponding focusing efficiency as high as $40 \%$ is demonstrated. Our designed dielectric metalens offers brilliant polarization insensitive operation over the entire defined UV spectrum because of the higher symmetry of the unit cells (and so the designed metalens). Moreover, the calculated full width at half maximum (FWHMs) of the focal spot is also near the diffraction limit. Our designed metalens will pave a new way towards the advancement of integration and miniaturization of the UV photonic systems and its applications. To the authors' knowledge, this is the first reported metalens in the UV regime with polarization independence and broadband (over $110 \mathrm{~nm}$ bandwidth) ability along with a high Numerical Aperture (NA) of 0.86 .

\section{Materials and Methods}

The optical properties of the selected material are very critical towards the development of a high-efficiency metasurface as specified by complex refractive index $\tilde{n}=n+i k$. The selected material should possess negligible absorption loss $(k=0)$ and a high refractive index $(n>2)$. Despite a negligible 
absorption, which is critical for high transmission efficiency, a higher refractive index promises strong confinement of the operating UV regime eventually providing full $0-2 \pi$ phase control. Traditional dielectric materials possess a narrow bandgap triggering high absorption losses in UV. Titanium oxide and gallium nitride have fairly large bandgaps, but they are not suitable to use due to their higher absorption losses at the targeted UV regime. $\mathrm{Si}_{3} \mathrm{~N}_{4}$ is chosen as a dielectric material for the UV metalens to realize high efficiency in UV spectral region. Due to its wide bandgap of about $5.1 \mathrm{eV}$, its large bandgap exhibits the wide transparency window by having exceptionally low extinction coefficient

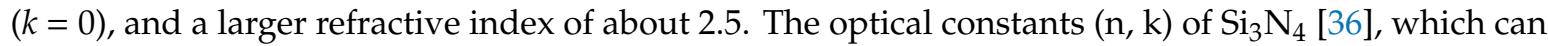
be considered as the fingerprint of the material, are shown in Figure S1.

There exist several options for considering the dielectric nanopillars for the design of metalenses, such as a slit, square cylinder, cuboid, cylinder, combined meta-molecules, and elliptic cylinders. These nanopillars could be divided into two kinds considering their sensitivity of polarization. The ones depending on the polarization of the light have rotation angles and are spatial anisotropic based on the Pancharatnam-Berry phase theory. Whereas the polarization insensitive are always square cylinders or cylinders utilizing side lengths or diameters to map the required phase. In this article, we use cylindrical nanopillars to control the phase of the light. The building blocks of the designed metalens are $\mathrm{Si}_{3} \mathrm{~N}_{4}$ nanopillars on a glass substrate shown in Figure 1a-c.
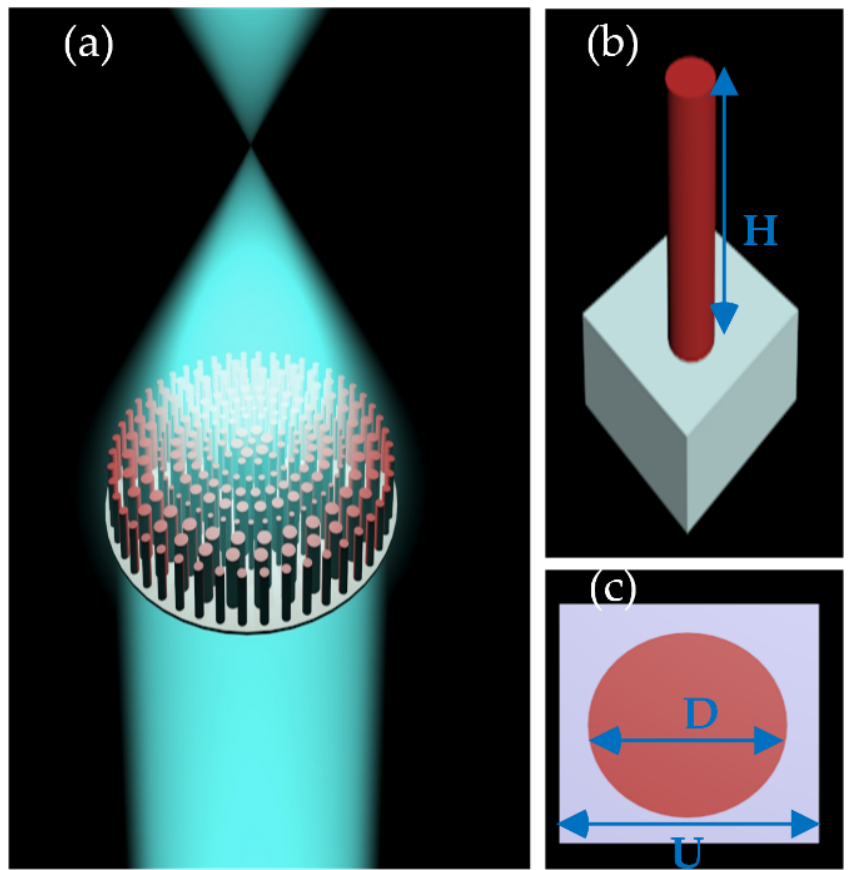

Figure 1. (a) Schematic view of the transmissive metalens. (b) Side view of the metalens building block. (c) Top of the metalens building block. For the designed wavelength of the metalens $\lambda=290 \mathrm{~nm}$, the nanopillar height $\mathrm{H}=600 \mathrm{~nm}$, unit cell dimension $\mathrm{P}=200 \mathrm{~nm}$, and the nanopillar radius varies between 30 and $92 \mathrm{~nm}$.

By optimizing the geometric parameters of the nanopillar full $2 \pi$ phase coverage can be realized. The unit cell dimension $(\mathrm{P})$, nanopillar radius $(\mathrm{R})$, and height $(\mathrm{H})$ are the three basic geometric and structural design parameters that control the phase and the transmission amplitude of the metasurface lens to ensure high efficiency. The design wavelength of the metalens is $290 \mathrm{~nm}$ and it is fully operational in the broadband spectrum of the UV light, i.e., $270-380 \mathrm{~nm}$ with excellent polarization insensitive behavior. Performing the simulations through commercial finite-difference-time-domain (FDTD) method implemented by commercial software 'FDTD Solutions' (produced by Lumerical Solutions Co. Ltd., Vancouver, BC, Canada) [37] the transmission and phase modulation of the nanopillar with different geometric parameters are calculated [35]. The nanopillar must have sufficient height 
to provide a full $2 \pi$ phase coverage over a range of radii. Besides, the unit cell dimension must be optimized wisely and the radius should be tuned in a manner to acquire the required phase profile. A set of simulations were performed to get the optimized values of the unit cell dimension, the height, and the range of the radius of nanopillar. Periodic boundary conditions were applied in $x$ and $y$ directions while perfectly matched layer (PML) boundary conditions were applied to the z-direction for calculating the aforesaid unit cell parameters.

\section{Design, Results, and Discussion}

The building blocks of the metalens are $\mathrm{Si}_{3} \mathrm{~N}_{4}$ nanopillars on the glass substrate. The metalens operates in transmission mode and it focuses the collimated light into a spot. To realize this, the required phase must be imparted by each nanopillar at the position $(x, y)$ as given by [38]

$$
\phi(x, y)=2 \pi-\frac{2 \pi}{\lambda_{d}}\left(\sqrt{x^{2}+y^{2}+f^{2}}-f\right)
$$

where $\lambda_{d}$ is the design wavelength and $f$ is the focal length. By varying the radius of nanopillars as a function of their position $(x, y)$ the effective index of the propagating mode is changed to achieve the desired phase profile $\phi(x, y)$ (Equation (1)). To achieve high transmission through the entire defined spectral range other parameters such as unit cell size $\mathrm{U}$ and the nanopillar height $\mathrm{H}$ are optimized at design wavelength $\lambda_{d}$. The optimized height of the nanopillar must be sufficiently tall so as to realize full $2 \pi$ phase coverage over a range of radius.

The optimized height of the nanopillar is $600 \mathrm{~nm}$, the radius of the $\mathrm{Si}_{3} \mathrm{~N}_{4}$ nanopillar varies between 30 and $92 \mathrm{~nm}$ besides, and the unit cell dimension is $200 \mathrm{~nm}$. The transmission and phase response calculated through the simulations at design wavelength $\lambda=290 \mathrm{~nm}, \mathrm{H}=600 \mathrm{~nm}$, and P $=200 \mathrm{~nm}$ are shown in Figure $2 \mathrm{a}, \mathrm{b}$, respectively. The transmission and phase response in the broadband region at $\mathrm{R}=36 \mathrm{~nm}$ can be seen in Figure 2c, d, respectively.
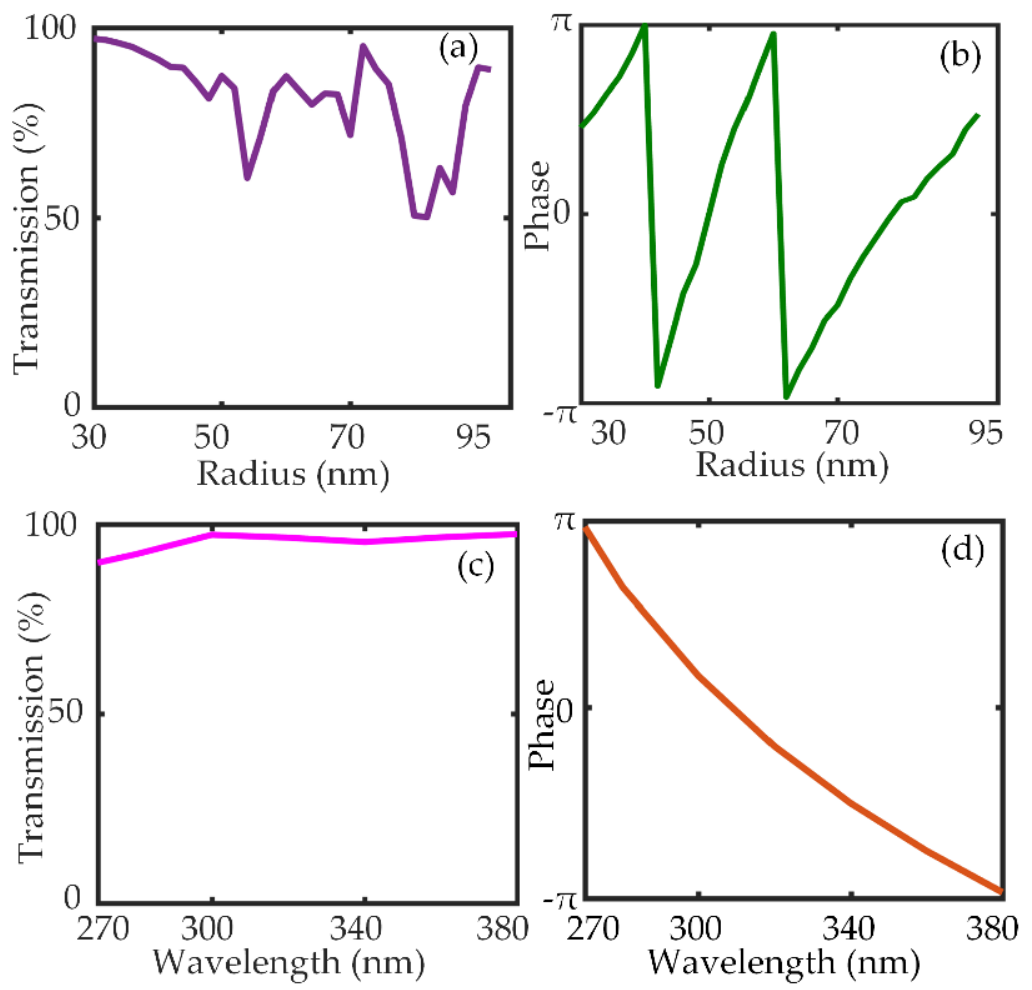

Figure 2. (a) Transmission as a function of radius. (b) Phase as a function of radius. (c) Transmission as a function of wavelength at $R=36 \mathrm{~nm}$. (d) Phase as a function of wavelength at $\mathrm{R}=36 \mathrm{~nm}$. 
It can be seen via the Figure 2 that the nanopillars are highly transmissive and can acquire the $2 \pi$ phase accumulation. To design the metalens, perfectly matched layer (PML) boundary conditions were applied in the $\mathrm{x}, \mathrm{y}$, and $\mathrm{z}$ directions. The required phase mask $\phi(x, y)$ was discretized by presuming square lattice unit cells with dimensions $\mathrm{U} \times \mathrm{U}$ so that the transmission error $\left|T_{m} e^{i \varphi_{t}(x i, y i)}-T(D) e^{i \varphi(D)}\right|[38]$ is minimized where $T_{m}$ is the averaged transmission. Figure 3 presents the focusing characteristics of the designed metalens. The NA of the metalens at the design wavelength $\lambda_{d}=290 \mathrm{~nm}$ is calculated to be 0.86 . The intensity profiles of the beam in the $x-z$ cross-section and $x-y$ cross-section of the metalens are shown in Figure 3.
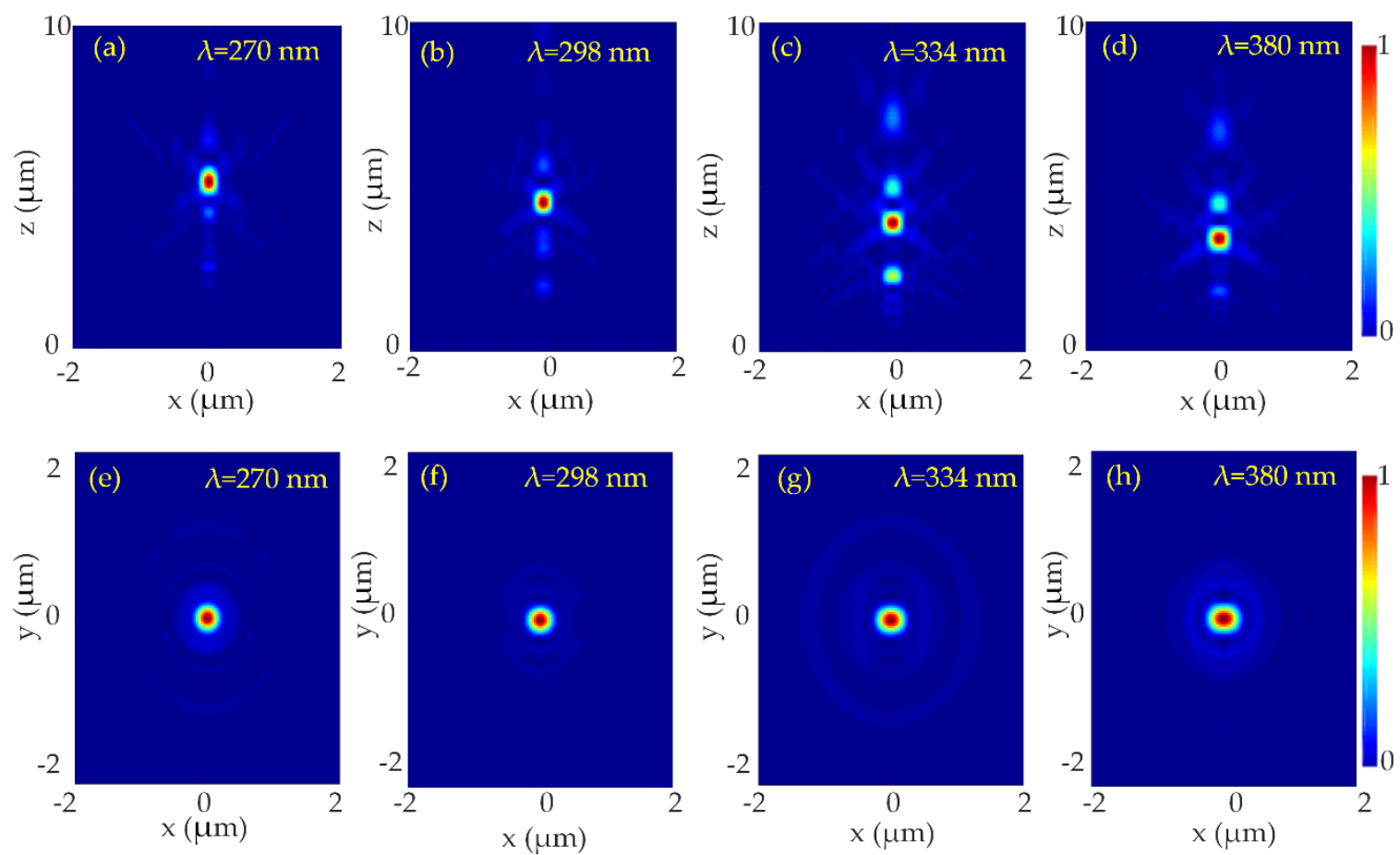

Figure 3. Normalized intensity distribution of the designed UV metalens at incident UV light 270-380 nm at the $x-z$ plane. (a) $\lambda=270 \mathrm{~nm}$, (b) $\lambda=298 \mathrm{~nm},(\mathbf{c}) \lambda=334 \mathrm{~nm}$ (d) $\lambda=380 \mathrm{~nm}$. The NA of the metalens at designed wavelength $\lambda_{d}=290 \mathrm{~nm}$ is 0.86 . Normalized intensity distribution of the designed UV metalens at incident UV light 270-380 nm at the $x-y$ plane at $x=y=0$. (e) $\lambda=270 \mathrm{~nm}$, (f) $\lambda=298 \mathrm{~nm},(\mathrm{~g}) \lambda=334 \mathrm{~nm}$ (h) $\lambda=380 \mathrm{~nm}$. The NA of the metalens at designed wavelength $\lambda_{d}=290 \mathrm{~nm}$ is 0.86 .

The intensity distribution of the focal spot shows a tightly focused, symmetric, and bright spot at the center of the focal plane at different wavelengths $(270 \mathrm{~nm}, 298 \mathrm{~nm}, 334 \mathrm{~nm}$, and $380 \mathrm{~nm})$. Due to the rotational symmetry of the designed metalens, focus spots for the TM and TE polarizations are just the same. Therefore, all the results are only given for TE polarization while omitting TM results.

The focal length of the metalens is calculated at representative wavelengths. At the focal points of the respective wavelengths i.e., $270 \mathrm{~nm}, 298 \mathrm{~nm}, 334 \mathrm{~nm}$, and $380 \mathrm{~nm}$, the FWHMs are calculated as $204 \mathrm{~nm}, 222 \mathrm{~nm}, 250 \mathrm{~nm}$, and $284 \mathrm{~nm}$. All the values are near diffraction-limited i.e., $\frac{\lambda_{d}}{2 \mathrm{NA}}$. The normalized intensity profiles of the focal spot in the $x$-direction (at $z=\mathrm{f}$, and $y=0$ ) are shown in Figure 4.

The focusing efficiency, which is defined as the ratio of the total light intensity at the focal spot to the transmitted light [8], is as high as $40 \%$. The focusing efficiency of the designed metalens is shown in Figure 5. 

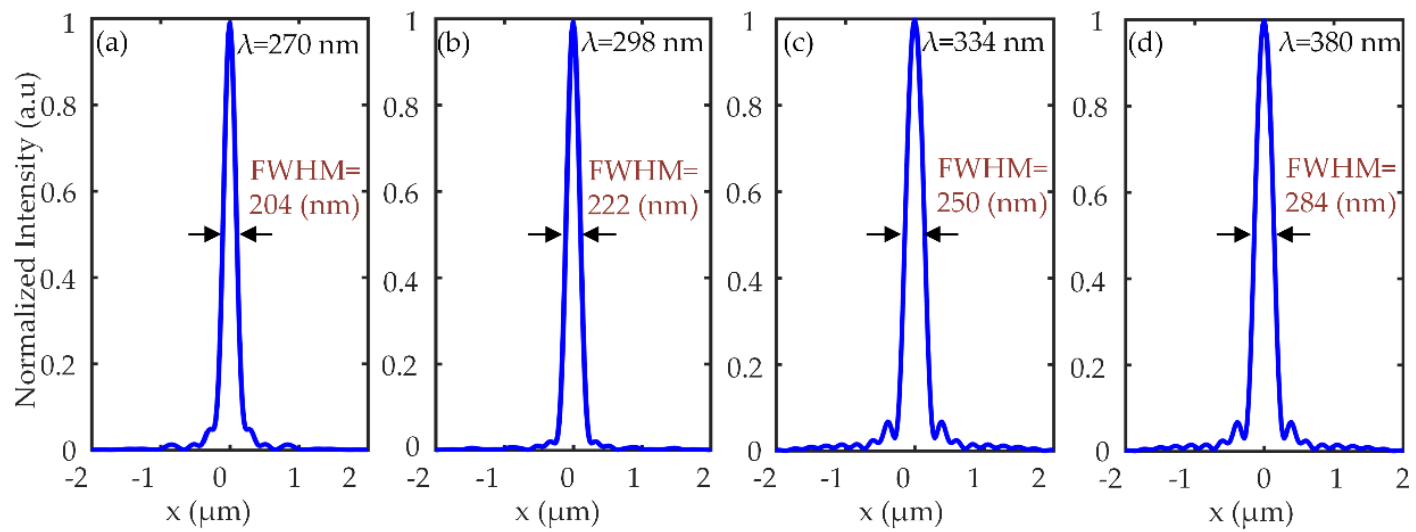

Figure 4. Normalized intensity profile of the focus spot along $x$-direction at respective wavelengths i.e., $270 \mathrm{~nm}, 298 \mathrm{~nm}, 334 \mathrm{~nm}$ and $380 \mathrm{~nm}$. (a) $\mathrm{y}=0, \mathrm{z}=5.3 \mu \mathrm{m}$, (b) $\mathrm{y}=0, \mathrm{z}=4.6 \mu \mathrm{m}$, (c) $\mathrm{y}=0, \mathrm{z}=4 \mu \mathrm{m}$ and (d) $\mathrm{y}=0, \mathrm{z}=3.1 \mu \mathrm{m}$.

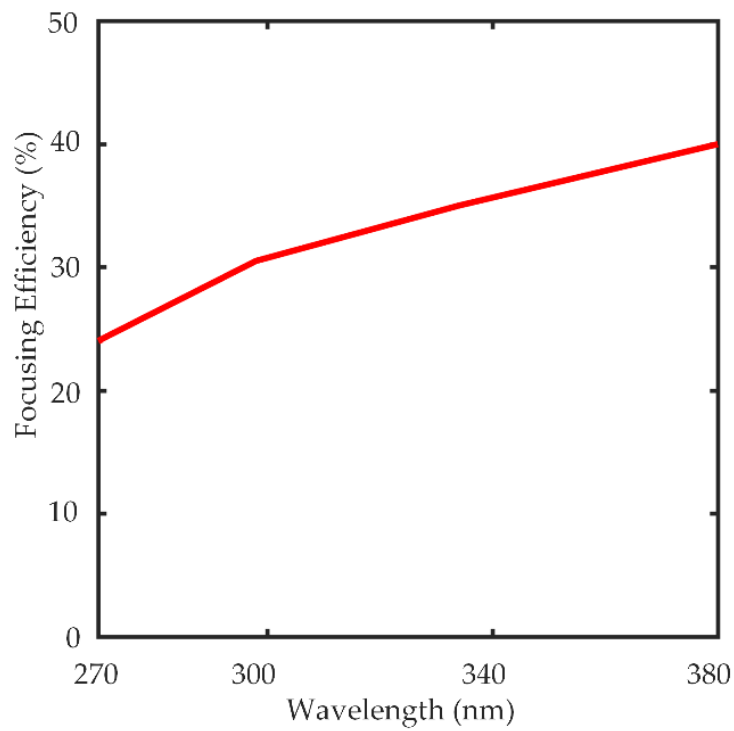

Figure 5. Focusing Efficiency of the metalens designed for the broadband UV spectrum 270-380 nm.

In the end, we compared our results with other references in terms of material platform, bandwidth, incident polarization, and NA; the comparison is shown in Table 1. We noticed that some of these previous studies are not polarization insensitive [33,34] while others are not broadband [29,32], and compared to these studies the NA of our designed metalens is the highest as well.

Table 1. Summary of our results and other references.

\begin{tabular}{ccccc}
\hline Reference & Material & $\begin{array}{c}\text { Wavelength } \\
(\mathbf{n m})\end{array}$ & Incident Light & NA \\
\hline Guo et al. [33] & $\mathrm{AlN}$ & $244-375$ & $\mathrm{CP}$ & $\mathrm{CP}$ \\
\hline Huang et al. [34] & $\mathrm{Nb}_{2} \mathrm{O}_{5}$ & 355 & Not mentioned \\
\hline Hu et al. [31] & $\mathrm{AlN}$ & $234-274$ & Polarization Insensitive & $>0.1$ \\
\hline Zhang et al. [29] & $\mathrm{HfO}_{2}$ & 325 & Polarization Insensitive & 0.6 \\
\hline Our Work & $\mathrm{Si}_{3} \mathrm{~N}_{4}$ & $270-380$ & Polarization Insensitive & 0.86 \\
\hline
\end{tabular}




\section{Conclusions}

This work reports on a high efficiency, polarization insensitive, near diffraction-limited UV metalens that operates in the broadband region of ultraviolet light from 270 to $380 \mathrm{~nm}$ with a tightly focused spot. The designed metalens is composed of silicon nitride cylindrical nanopillars arranged on a square lattice. The limitations of the plasmonics metalenses, such as Ohmic losses, are overcome by using dielectric silicon nitride nanopillars as the basic building blocks of the metalens. By altering the geometric parameters of the unit cell, high transmission, and sufficient phase coverage is achieved in the target wavelength range. The focusing efficiency is calculated to be as high as $40 \%$. The designed high-efficiency UV metalens can be used in lightweight, low cost, and planar UV devices, such as UV router, lithography, image sensor, UV laser, communication and sterilization, and so on. Achieving a high-efficiency polarization insensitive metalens operating in the broadband UV range is a critical step toward the implementation of the metasurfaces for practical applications in nanophotonics and integrated optics in the UV. This should promote the high-density integration and miniaturization of the UV nanophotonics. In the future, we intend to maximize the focusing efficiency and design an achromatic, broadband UV metalens to incorporate them into daily life, for widespread applications.

Supplementary Materials: The following are available online at http://www.mdpi.com/2079-4991/10/8/1439/s1, Figure S1: The real and imaginary part of the refractive index of $\mathrm{Si}_{3} \mathrm{~N}_{4}$ utilized in this study, displaying a high refractive index $(n>2)$ and exceptionally low loss $(k=0)$ over a broad UV range.

Author Contributions: S.K. perceived and designed the metalens and also wrote the original manuscript; B.Y., X.C., D.K., Y.K., C.B., and S.U. backed the analysis; J.W. and D.Z. supervised the study and reviewed the manuscript. All authors have read and agreed to the published version of the manuscript.

Funding: This work is supported by National Science Foundation Project 61775140 and 81701745, National Key R\&D Program of China 2018YFA0701800, Shanghai Municipal Science and Technology Commission Innovation Action Plan 18DZ1100400, Joint Funds of the National Natural Science Foundation of China U1831133.

Conflicts of Interest: The authors declare no conflict of interest.

\section{References}

1. Lin, B.-J. Immersion lithography and its impact on semiconductor manufacturing. J. Micro/Nanolithogr. MOEMS 2004, 3, 377-501. [CrossRef]

2. Abdollahramezani, S.; Taghinejad, H.; Fan, T.; Kiarashinejad, Y.; Eftekhar, A.A.; Adibi, A. Reconfigurable multifunctional metasurfaces employing hybrid phase-change plasmonic architecture. arXiv 2018, arXiv:1809.08907. Available online: https://arxiv.org/abs/1809.08907 (accessed on 15 February 2020).

3. Zhang, M.; Pu, M.; Zhang, F.; Guo, Y.; He, Q.; Ma, X.; Huang, Y.; Li, X.; Yu, H.; Luo, X. Plasmonic metasurfaces for switchable photonic spin—Orbit interactions based on phase change materials. Adv. Sci. 2018, 5, 1800835. [CrossRef] [PubMed]

4. Wen, J.; Feng, H.; Chen, J.; Wang, K.; Lv, Y.; Zhong, Y.; Zhang, D. Plasmonic holographic metasurfaces for generation of vector optical beams. IEEE Photonics J. 2017, 9, 1-8. [CrossRef]

5. Merlin, R. Radiationless electromagnetic interference: Evanescent-field lenses and perfect focusing. Science 2007, 317, 927-929. [CrossRef] [PubMed]

6. Khorasaninejad, M.; Ambrosio, A.; Kanhaiya, P.; Capasso, F. Broadband and chiral binary dielectric meta-holograms. Sci. Adv. 2016, 2, e1501258. [CrossRef] [PubMed]

7. Bai, W.; Yang, P.; Huang, J.; Chen, D.; Zhang, J.; Zhang, Z.; Yang, J.; Xu, B. Near-infrared tunable metalens based on phase change material Ge 2 Se 2 Te 5. Sci. Rep. 2019, 9, 1-9. [CrossRef]

8. Sun, S.; Zhou, Z.; Zhang, C.; Gao, Y.; Duan, Z.; Xiao, S.; Song, Q. All-dielectric full-color printing with $\mathrm{TiO}_{2}$ metasurfaces. ACS Nano 2017, 11, 4445-4452. [CrossRef]

9. Wang, S.; Wu, P.C.; Su, V.-C.; Lai, Y.-C.; Chen, M.-K.; Kuo, H.Y.; Chen, B.H.; Chen, Y.H.; Huang, T.-T.; Wang, J.-H. A broadband achromatic metalens in the visible. Nat. Nanotechnol. 2018, 13, 227-232. [CrossRef]

10. Maguid, E.; Yulevich, I.; Yannai, M.; Kleiner, V.; Brongersma, M.L.; Hasman, E. Multifunctional interleaved geometric-phase dielectric metasurfaces. Light Sci. Appl. 2017, 6, e17027. [CrossRef] 
11. Chen, M.K.; Wu, P.C.; Tsai, W.-Y.; Wang, H.-C.; Chen, J.-W.; Liao, C.Y.; Chu, C.H.; Sun, G.; Tsai, D.P. Versatile Polarization Generation by Using Aluminum Plasmonic Metasurface. In Proceedings of the Frontiers in Optics, Washington, DC, USA, 18-21 September 2017; p. JW3A. 110.

12. Arbabi, A.; Horie, Y.; Bagheri, M.; Faraon, A. Dielectric metasurfaces for complete control of phase and polarization with subwavelength spatial resolution and high transmission. Nat. Nanotechnol. 2015, 10, 937. [CrossRef]

13. Ahmadivand, A.; Semmlinger, M.; Dong, L.; Gerislioglu, B.; Nordlander, P.; Halas, N.J. Toroidal dipole-enhanced third harmonic generation of deep ultraviolet light using plasmonic meta-atoms. Nano Lett. 2018, 19, 605-611. [CrossRef]

14. Ahmadivand, A.; Gerislioglu, B.; Ramezani, Z. Gated graphene island-enabled tunable charge transfer plasmon terahertz metamodulator. Nanoscale 2019, 11, 8091-8095. [CrossRef] [PubMed]

15. Bai, W.; Yang, P.; Wang, S.; Huang, J.; Chen, D.; Zhang, Z.; Yang, J.; Xu, B. Tunable duplex metalens based on phase-change materials in communication range. Nanomaterials 2019, 9, 993. [CrossRef] [PubMed]

16. Yu, B.; Wen, J.; Chen, X.; Zhang, D. An achromatic metalens in the near-infrared region with an array based on a single nano-rod unit. Appl. Phys. Express 2019, 12, 092003. [CrossRef]

17. Jahani, S.; Jacob, Z. All-dielectric metamaterials. Nat. Nanotechnol. 2016, 11, 23. [CrossRef]

18. Yu, N.; Capasso, F. Flat optics with designer metasurfaces. Nat. Mater. 2014, 13, 139-150. [CrossRef]

19. Li, L.; Cui, T.J.; Ji, W.; Liu, S.; Ding, J.; Wan, X.; Li, Y.B.; Jiang, M.; Qiu, C.-W.; Zhang, S. Electromagnetic reprogrammable coding-metasurface holograms. Nat. Commun. 2017, 8, 1-7. [CrossRef]

20. Choudhury, S.M.; Wang, D.; Chaudhuri, K.; DeVault, C.; Kildishev, A.V.; Boltasseva, A.; Shalaev, V.M. Material platforms for optical metasurfaces. Nanophotonics 2018, 7, 959-987. [CrossRef]

21. Kiarashinejad, Y.; Abdollahramezani, S.; Adibi, A. Deep learning approach based on dimensionality reduction for designing electromagnetic nanostructures. npj Comput. Mater. 2020, 6, 1-12. [CrossRef]

22. Yang, H.; Cao, G.; Shang, X.; Li, T.; Yang, G.; Li, G. Anisotropic metasurfaces for efficient polarization independent wavefront steering. J. Phys. D Appl. Phys. 2019, 53, 045104. [CrossRef]

23. Arbabi, A.; Faraon, A. Fundamental limits of ultrathin metasurfaces. Sci. Rep. 2017, 7, 43722. [CrossRef] [PubMed]

24. Hong, X.; Feng, S.; Guo, H.; Li, C. A beam deflector with dielectric metasurfaces in the terahertz region. Laser Phys. 2019, 30, 016204. [CrossRef]

25. Yu, B.; Wen, J.; Chen, L.; Zhang, L.; Fan, Y.; Dai, B.; Kanwal, S.; Lei, D.; Zhang, D. Polarization-independent highly efficient generation of Airy optical beams with dielectric metasurfaces. Photonics Res. 2020, 8, 1148-1154. [CrossRef]

26. Proust, J.; Bedu, F.; Gallas, B.; Ozerov, I.; Bonod, N. All-dielectric colored metasurfaces with silicon Mie resonators. ACS Nano 2016, 10, 7761-7767. [CrossRef] [PubMed]

27. Khorasaninejad, M.; Shi, Z.; Zhu, A.Y.; Chen, W.-T.; Sanjeev, V.; Zaidi, A.; Capasso, F. Achromatic metalens over $60 \mathrm{~nm}$ bandwidth in the visible and metalens with reverse chromatic dispersion. Nano Lett. 2017, 17, 1819-1824. [CrossRef]

28. Ollanik, A.J.; Smith, J.A.; Belue, M.J.; Escarra, M.D. High-efficiency all-dielectric Huygens metasurfaces from the ultraviolet to the infrared. ACS Photonics 2018, 5, 1351-1358. [CrossRef]

29. Zhang, C.; Divitt, S.; Fan, Q.; Zhu, W.; Agrawal, A.; Lu, Y.; Xu, T.; Lezec, H.J. Low-loss metasurface optics down to the deep ultraviolet region. Light Sci. Appl. 2020, 9, 1-10. [CrossRef]

30. Ahmed, H.; Rahim, A.A.; Maab, H.; Ali, M.M.; Naureen, S. Polarization insensitive all-dielectric metasurfaces for the ultraviolet domain. Opt. Mater. Express 2020, 10, 1083-1091. [CrossRef]

31. Hu, M.; Wei, Y.; Cai, H.; Cai, Y. Polarization-insensitive and achromatic metalens at ultraviolet wavelengths. J. Nanophotonics 2019, 13, 036015. [CrossRef]

32. Zhang, C.; Divitt, S.; Fan, Q.; Zhu, W.; Agrawal, A.; Xu, T.; Lezec, H.J. All-dielectric deep ultraviolet metasurfaces. In Proceedings of the CLEO: QELS_Fundamental Science, San Jose, CA, USA, 5-10 May 2019; p. FM3C. 3.

33. Guo, L.; Xu, S.; Wan, R.; Li, T.; Xiong, L.; Wang, L.; Zhu, W. Design of aluminum nitride metalens in the ultraviolet spectrum. J. Nanophotonics 2018, 12, 043513.

34. Huang, K.; Deng, J.; Leong, H.S.; Yap, S.L.K.; Yang, R.B.; Teng, J.; Liu, H. Ultraviolet Metasurfaces of $\approx 80 \%$ Efficiency with Antiferromagnetic Resonances for Optical Vectorial Anti-Counterfeiting. Laser Photonics Rev. 2019, 13, 1800289. [CrossRef] 
35. Kanwal, S.; Wen, J.; Yu, B.; Kumar, D.; Chen, X.; Kang, Y.; Bai, C.; Zhang, D. High-Efficiency, Broadband, Near Diffraction-Limited, Dielectric Metalens in Ultraviolet Spectrum. Nanomaterials 2020, 10, 490. [CrossRef]

36. Palik, E.D.; Ghosh, G. Electronic Handbook of Optical Constants of Solids: User Guide; Academic Press: Cambridge, MA, USA, 1999.

37. Khorasaninejad, M.; Chen, W.T.; Devlin, R.C.; Oh, J.; Zhu, A.Y.; Capasso, F. Metalenses at visible wavelengths: Diffraction-limited focusing and subwavelength resolution imaging. Science 2016, 352, 1190-1194. [CrossRef] [PubMed]

38. Khorasaninejad, M.; Zhu, A.Y.; Roques-Carmes, C.; Chen, W.T.; Oh, J.; Mishra, I.; Devlin, R.C.; Capasso, F. Polarization-insensitive metalenses at visible wavelengths. Nano Lett. 2016, 16, 7229-7234. [CrossRef]

(C) 2020 by the authors. Licensee MDPI, Basel, Switzerland. This article is an open access article distributed under the terms and conditions of the Creative Commons Attribution (CC BY) license (http://creativecommons.org/licenses/by/4.0/). 